\title{
Zur Erneuerung des Geographieunterrichtes in der Schweiz (II)
}

\section{Laufende Arbeiten an Lehrplänen}

Das Projekt "Schule und Dritte Welt" am Insti tut für Unterrichtsfragen und Lehrerfortbildung Basel

Das Projekt "Schule und Dritte Welt" am Institut für Unterrichtsfragen und Lehrerfortbildung Basel (ULEF) läuft seit einem Jahr. Einerseits ist dem Lehrer zu zeigen, dass die Beziehungen zwischen den Industrieländern und den Entwicklungsländern und die allgemeinen Probleme der Dritten Welt von vorrangiger Bedeutung insbesondere im Geographieunterricht sind. Anderseits sollen Arbeitsunterlagen für den Unterricht über dieses Thema bereitgestellt und methodisch-didaktische Hinweise gegeben werden.

In einer zweisemestrigen Arbeitsgemeinschaft, die für Geographie- und Geschichtslehrer der Mittel- und Oberstufe offen stand, wurde vorerst versucht, ein Curriculum zu entwerfen. Die Resultate werden in einem Arbeitsbericht zusammengefasst. Unter anderem zeigte sich, dass der Lehrer beim richtigen Einbau des Themas in seinen Unterricht auf mannigfaltige Schwierigkeiten inhaltlicher, vor allem aber auch lernpsychologischer Art stossen wird. Im Wintersemester 1974/75 laufen zwei Kurse: Im ersten werden audiovisuelle Medien evaluiert, im zweiten Unterrichtseinheiten über ausgewählte Entwicklungsländer und entsprechende allgemeine Probleme erarbeitet. - In einer späteren Phase soll für die Sekundarstufe 1 ein entsprechendes Curriculum entworfen werden. Gleichzeitig stellt die Pädagogische Dokumentationsstelle Basel alle verfügbaren Materialien zusammen, und im Basler Schulblatt erscheinen vermehrt Rezensionen über Lehrmittel, die die Dritte Welt behandeln. Damit soll der Lehrer auf die Thematik sensibilisiert werden, die nur ein Teilaspekt ist von dem, was man vielleicht als Umweltskunde bezeichnen könnte.

Die erneuerten Geographielehrpläne an den Realschulen des Kantons Baselland (6. -10 . Schuljahr)

1970 erschien ein pragmatisch revidierter Entwurf einer neuen Stundentafel für die allgemeine Abteilung der Realschulen von Baselland. Er sah unter anderem vor, den obligatorischen Geographieunterricht um 3 Jahre zu reduzieren und ihn innerhalb dieser Reduktion während zweier Jahre als Wahlfachkurs auszugest alten. Die Lehrerschaft wurde verunsichert. Da reagierte eine Gruppe Geographie-Fachlehrer durch die Einberufung einer "Fachkonferenz der Geographielehrer von Baselland". Diese Fachkonferenz hat seither die Revision der Lehrpläne durchgeführt und gleichzeitig für die einzelnen Jahrespensen Lehrhilfen oder "Geographie-Kataloge" geschaffen. Diese sind als Präparationshilfe gedacht und enthalten Stoffgliederungen mit den entsprechenden Angaben über Literatur und Anschauungsmaterialien. Vier Hefte liegen bereits vor, und der Geographiekatalog für die fünfte Klasse (10. Schuljahr) erscheint demnächst.

Die Revisionsarbeit an den Unterrichtsgrundlagen wurde begleitet durch eine Pressekampagne, in der allgemein für die Geographie geworben wurde. Dadurch konnte die Oeffentlichkeit sensibilisiert werden und es gelang, die von der "Fachkonferenz der Geographielehrer Baselland" revidierten Lehrpläne und die neuen Geographie-Kataloge durch den kantonalen Erziehungsrat genehmigen zu lassen.

Literatur: Geographie Katalog (Lehrhilfe) zum revidierten Lehrplan 1971, 5 Hefte

(1. - 5. Klasse Reals chule).

Bezug: Schulinspektorat Baselland, Liestal.

Die Schweiz und die Welt im Wandel Eine Arbeitshilfe für den Geographieunterricht im 8. oder 9. Schuljahr.

1972 erschien im Kanton Bern ein neuer Lehrplan für die Primarschulen. Er enthält fachweise Angaben über Ziel, Allgemeine Bestimmungen und Stoff, für die Geographie insgesamt 2, 5 Druckseiten. Im Unterricht am Lehrerseminar Hofwil-Bern zeigte sich in einem Geographie und Methodik umfassenden Unterrichtsprojekt, dass die im Lehrplan enthaltenen Angaben für den Junglehrer zu dürftig sind. Anschliessend konnte in einem Lehrerweiterbildungskurs für Primar- und Sekundarlehrer eine Arbeitshilfe zum Thema "Die Schweiz und die Welt im Wandel" entworfen werden, die sowohl das Interesse des Delegierten für Raumplanung (Prof. M. Rotach) wie dasjenige der Kantonalen Erziehungsdirektion (Amt für Unterrichtsforschung) fand. In einem weiteren, nun 
ganzjährigen Lehrerkurs wird im Schuljahr 1974/75 der Entwurf im Unterricht ausprobiert. Im März 1975 erscheint die bereinigte Fassung gleichzeitig im Staatlichen Lehrmittelverlag Bern und im Geographischen Institut der Universität Bern (GEOGRAPHICA BERNENSIA Heft S 1).

Die Arbeitshilfe besteht aus vierspaltigen Tabellen, die abschnittweise Lernziel (Verhaltens ziele), Stoffziel, Methodische Bemerkungen und Hinweise auf Literatur, Hilfsmittel und Querverbindungen enthalten.

Inhaltlich gliedert sie sich in die Problemkreise Wandel im Landschaftsbild, Landwirtschaft; Industrie, Beispiele aus dem Dienstleistungssektor, Bevölkerung und Siedlungsbild, Beispie le aus der Raumplanung. Dabei wird zwischen Grundstoff (Fundamentum) und Ergänzungsstoff unterschieden, innerhalb dessen der Lehrer seine regional oder lokal bedingten Schwerpunkte wählen kann. Die präzise Zielumschreibung erlaubt es, die Arbeitshilfe in jedem Regionalraum anzuwenden.

Literaturangaben (Minimalliste, statistische Literatur, Regionalliste für den Raum Bern, Ergänzungsliste) und Materialkataloge (Verbrauchsmaterial, Schulsammlung) sowie Hinweise auf die durch den Lehrer zu treffenden Vorbereitungen (Teamarbeit) ergänzen die Darstellung.

Die geschilderte Erneuerungsarbeit charakterisiert sich durch die kooperative Arbeit von Lehrern verschiedener Schulstufen (Universität, Lehrerseminar, Sekundar-und Primarschule) und bildet das Beispiel einer lehrerzentrierten Curriculumreform, die ihren Ansatz in der Erneuerung bestehender Lehrpläne gefunden hat.

Literatur: Die Schweiz und die Welt im Wandel, Staatlicher Lehrmittelverlag Bern und Geographisches Institut der Universität Bern (Geographica Bernensia Heft S 1).

\section{Neue Lehrmittel}

Der Einsatz des Schulwandbildes auf der Mittelschulstufe

Seit Jahrzehnten gibt der Schweiz. Lehrerverein sogenannte Schulwandbilder heraus. Darunter finden wir viele geographische Themen: Fjord, Vulkan, Delta, Oase, Jura, Karst, Glet- scher usw. Diese Bilder, meist von namhaften Künstlern geschaffen, werden im Geographieunterricht der Mittelschulstufe eingesetzt, sie entsprechen den Bedürfnissen eines modernen Unterrichts kaum.

Seit 1974 erscheinen nun im Rahmen der geographischen Bilder photographische Luftaufnahmen der Swissair. 1974 hiess das Thema " "Disentis und das Bündner Oberland", 1975 "Spreitenbach - vom Dorf zur Stadt" und "Allaman - ein waadtländisches Winzerdörfchen". Die neue Reihe verspricht für den Unterricht dann wertvoll zu werden, wenn ein klares Konzept sichtbar wird, wenn verschiedene Landschaften, Siedlungs - und Wirtschaftsformen - verglichen und einander gegenübergestellt werden können. In der vorliegenden Form eignen sich die Bilder in erster Linie für den Gruppenunterricht und für Einzelaufgaben. Die Besprechung im Klassenverband könnte man sich dann vorstellen, wenn die Bilder den Schülern im Kleinformat - A3 oder A4 - vorgelegt werden könnten.

An der Fortsetzung dieser Luftbildreihe sind wir interessiert. Die dazugehörenden, sorgfältig geschriebenen Kommentarhefte zeigen, dass die Bilder auch auf der Mittelschulstufe zur Unterrichtsgestaltung beitragen können.

Unterrichtswerk "Geographie" von Klett Das Informationsbüro Zug des Ernst Klett Verlages hat im vergangenen Sommer interessierte Kreise zu einem Referat über das Thema "Geographie-Unterricht nach neuen Lehrplänen" eingeladen. Dabei ging es in erster Linie um das Bekanntmachen eines multimedialen Geographie-Unterrichtswerkes für die Sekundarstufe I (5. -10. Schuljahr). Hauptbestandteil dieses Werkes sind 3 reich illustrierte Schülerbände, denen umfangreiches Hilfsmaterial zugeordnet ist, wie zum Beispiel Lesehefte, Arbeitsblätter, Lehrerringbücher mit didaktischen Hinweisen, Arbeitstransparente mit Schemata, Karten und Senkrechtaufnahmen ausgewählter Typlandschaften Deutschlands, sowie Diareihen und Landschaftsquerschnitte. Zum Teil sind den Schweizer Geographielehrern diese Ergänzungsmaterialien aus dem Klett-Verlag bereits seit längerer Zeit bekannt, aber sie bilden jetzt zusammen mit den Neuerscheinungen ein Ganzes. Auffal- 
lendstes Merkmal der 3 Schülerbände, die den Lehrgang weitgehend bestimmen, ist die vollständige Abkehr von der Länderkunde zugunsten einer "allgemeinen Geographie an Beispielen". Die Begründung liegt darin, dass sich nur allgemeingeographisch-exemplarischer Unterricht auf Lernziele ausrichten lässt, nicht aber der Länderkundeunterricht, der ja eigentlich nur einzelnen Regionen gerecht wird. Damit kommt das Werk dem Bedürfnis, im Geographieunterricht nicht vorwiegend enzyklopädisches Wissen zu vermitteln, sondern die Schüler zu transferierbarem Können zu befähigen, in hohem Masse entgegen. Auch der alte Grundsatz "vom Nahen zum Fernen" wurde fallen gelassen. An seiner Stelle tritt ein Fortschreiten von einfachen zu komplizierten Strukturen, die auf die Interessenslage und Altersstufe abgestimmt sind.

Die vorliegende Trilogie mit den sie begleitenden Lehrerkommentaren ist ein Beispiel, wie die Unterrichtserneuerung in der Bundesrepublik Deutschland nach dem Vorliegen des Grundsatzpapiers "Zur Gestaltung und Zielsetzüng geographischen Unterrichts" 1970 eingesetzt hat.

Literatur: Geographie 5. und 6. Schuljahr Geographie 7. und 8. Schuljahr, Geographie 9. und 10. Schuljahr; Arbeitsmaterialien Geographie - Schülerarbeitsblätter Mappe 1 und 2 (5. und 6. Schuljahr); Elemente zur Unterrichtsplanung Band 1 und 2; alle Verlag Klett, Stuttgart.

\section{Zusammenfassung und Ausblick}

Die den Referaten folgende Diskussion verdeutlichte folgende Gesichtspunkte:

- Eine zeitgemässe Erneuerung des Geographieunterrichtes hat eingesetzt. Sie wird von den Lehrern her als lehrerzentrierte Curriculumreform aufgebaut und kann auf verständnisvolle Mitarbeit von Behörden, Vertretern der Wirtschaft und der Planung sowie von Museen rechnen.

- Der Ansatz zu Reformen liegt in der Regel bei bestehenden Lehrplänen, die schrittweise umgebaut werden. Dadurch wird ermöglicht, dass Bewährtes eingebaut werden kann, wobei freilich mit dem Verzicht auf einen radikalen Neubeginn Gesamterneue- rungen nur langfristig realisiert werden können.

Lehrmittel sollten nicht mehr nur aus einem Textbuch für den Schüler bestehen, sondern aus verschiedenen Teilen wie Lehrerhandbuch, Schülerbuch, Arbeitsblättern, Transparentfolien, Diasammlung und zugehörigem Kommentar.

Im Unterschied zur Entwicklung in der Bundesrepublik Deutschland richtet sich die Geographie auf der Volkss chulstufe noch beinahe ausschliesslich nach dem Prinzip "von der Nähe in die Ferne". Die auf das Prinzip der Anschauung zurückgehende Idee hat aber seit dem Aufkommen der Massenmedien die Bedeutung als absolute Richtlinie verloren.

Die Entwicklung neuer Lehrpläne oder Lehrmittel in der Schweiz erfolgt noch zu oft in isoliertem Rahmen ausschliesslich für einen Kanton oder für eine bestimmte Schulstufe. Löbliche Ausnahmen sind zu verzeichnen. In diesem Bereich erwächst dem Schweizerischen Geographielehrer verein eine besondere Aufgabe, liessen sich doch durch seine auf verschiedenen Schulstufen tätigen Mitglieder regionale Arbeitskreise bilden, welche die anzustrebende vertikale und horizontale Integration in den kommenden Erneuerungsarbeiten durchführen könnten. 